\title{
Minimizing the weighted sum of completion times under processing time uncertainty
}

\author{
Zacharie ALES ${ }^{2}$ \\ Laboratoire Informatique d'Avignon \\ University of Avignon \\ 84911 Avignon, France \\ Thi Sang NGUYEN ${ }^{3}$ \\ International Francophone Institute \\ C3- 144, Xuan Thuy, Hanoi, Vietnam \\ Michael POSS ${ }^{1}$ \\ UMR CNRS 5506 LIRMM, Université de Montpellier, 161 rue Ada, 34392 \\ Montpellier Cedex 5, France
}

\begin{abstract}
We address the robust counterpart of a classical single machine scheduling problem by considering a budgeted uncertainty and an ellipsoidal uncertainty. We prove that the problem is $\mathcal{N} \mathcal{P}$-hard for arbitrary ellipsoidal uncertainty sets. Then, a mixedinteger linear programming reformulations and a second order cone programming reformulations are provided. We assess the reformulations on randomly generated instances, comparing them with branch-and-cut algorithms.
\end{abstract}

Keywords: Integer programming, robust optimization, scheduling. 


\section{Introduction}

Scheduling is a rich topic within combinatorial optimization that has witnessed a large amount of research in the past decades, including applications oriented works, integer programming formulations, polyhedral studies, and approximation algorithms. In this work, we focus in this work on one of the simplest scheduling problem, which can be defined as follows. We are given a set $\mathcal{J}=\{1, \ldots, n\}$ of jobs, each having a processing time $p_{j}$ and a weight $w_{j}$, and we would like to order the jobs so as to minimize the weighted sum of their completion times. Formally, letting $\sigma(i)$ be the position of job $i$ for the permutation $\sigma$ and $C_{j}(\sigma)=\sum_{i=1}^{\sigma(j)} p_{\sigma^{-1}(i)}$ be the completion time of job $j$ for permutation $\sigma$, we want to solve the optimization problem

$$
\min _{\sigma \in P(n)} \sum_{j \in \mathcal{J}} w_{j} C_{j}(\sigma)
$$

where $P(n)$ represents the set of permutations of $\{1, \ldots, n\}$. It is well-known that Problem (1) can be solved in polynomial time by ordering the jobs according to their non-decreasing value of $p_{j} / w_{j}$, which is known as Smith's rule [12].

In practical scheduling problems, the parameters of the problem are usually subject to variations, and this is particularly true for the vector of processing times $p$, whose value can be affected by various hazardous events, such as machine breakdowns, working environment changes, worker performance instabilities, to cite a few. We address this issue herein through the lens of min max robust optimization. Specifically, we assume that the uncertainty over $p$ is characterized by a given convex set $U \subset \mathbb{R}^{n}$, and we study the robust counterpart of (1) that is defined as

$$
\min _{\sigma \in P(n)} \max _{p \in U} \sum_{j \in \mathcal{J}} w_{j} C_{j}(\sigma, p)
$$

where $C_{j}(\sigma, p)=\sum_{i=1}^{\sigma(j)} p_{\sigma^{-1}(i)}$ denotes the completion time of job $j$ for permutation $\sigma$ and the vector of processing times taking value $p$.

For arbitrary uncertainty sets $U$, it is well known that Problem (2) is $\mathcal{N P}$-hard, see [14], even when $w_{j}=1$ for each $j \in \mathcal{J}$ and $U$ is the convex

1 Email: michael.poss@lirmm.fr

2 Email: zacharie.ales@univ-avignon.fr

3 Email: ntsang@ifi.edu.vn 
hull of two vectors. This is not a surprising result, as it is well-known that robust combinatorial optimization problems with arbitrary uncertainty sets are, more often than not, harder than their deterministic counterparts [1]. While arbitrary uncertainty sets offer little hope for efficient algorithmic solutions, Bertsimas and Sim [4] have proposed a specific uncertainty set that preserves the complexity of many robust combinatorial optimization problems. In our context, their set can be defined as follows. Given two positive vectors $\bar{p}$ and $\hat{p}$ that respectively represent the nominal value of and the deviation of $p$, and a positive integer $\Gamma$, we consider the set

$$
U^{\Gamma} \equiv\left\{p \in \mathbb{R}^{n}: p_{j}=\bar{p}_{j}+\delta_{j} \hat{p}_{j}, j \in \mathcal{J}, \delta \in\{0,1\}^{n}, j \in \mathcal{J}, \sum_{j \in \mathcal{J}} \delta_{j} \leq \Gamma\right\} .
$$

Following the notations of $[6,9]$, let us denote Problem (2) for set $U^{\Gamma}$ by 1||$U_{p}^{\Gamma} \mid \sum_{j} w_{j} C_{j}$. When $w_{j}=1$ for each $j \in \mathcal{J}$, which is denoted 1||$U_{p}^{\Gamma} \mid \sum_{j} C_{j}$, Bougeret et al. [6] have proved that the problem can be solved in $O\left(n^{5}\right)$. Tadayon and Smith [13] have proposed a faster algorithm when $\hat{p}_{j}=\kappa \bar{p}_{j}$ for some $\kappa>0$, running in $O(n \log n)$. On the negative side, the problem is $\mathcal{N P}$-hard in the strong sense for arbitrary weights [6].

The hardness result of [6] was the initial motivation for the current work. Specifically, our first contribution is to provide a preliminary mixed-integer linear programming study of 1||$U_{p}^{\Gamma} \mid \sum_{j} w_{j} C_{j}$. Our second contribution concerns Problem 1||$U_{p}^{\Omega} \mid \sum_{j} w_{j} C_{j}$, which is defined as Problem (2) when considering the ellipsoidal uncertainty set defined by the positive definite matrix $\Sigma \in \mathbb{R}^{n \times n}$

$$
U^{\Omega} \equiv\left\{p \in \mathbb{R}^{n}: p_{j}=\bar{p}_{j}+\delta_{j} \hat{p}_{j}, j \in \mathcal{J},\left\|\Sigma^{-\frac{1}{2}} \delta\right\|_{2} \leq \Omega\right\} .
$$

We prove that 1||$U_{p}^{\Omega} \mid \sum_{j} w_{j} C_{j}$ is $\mathcal{N} \mathcal{P}$-hard and assess its numerical difficulty through mixed-integer second order cone programming reformulations. The hardness result is provided in Section 2. Section 3 presents our integer programming formulations and Section 4 reports our numerical experiments.

\section{Complexity of 1||$U_{p}^{\Omega} \mid \sum_{j} w_{j} C_{j}$}

Suppose that $w_{j}=1$ for each $j \in \mathcal{J}$. Given any permutation $\sigma \in P(n)$, notice that

$$
\sum_{j \in \mathcal{J}} C_{j}(\sigma, p)=\sum_{j \in \mathcal{J}}(n+1-\sigma(j)) p_{j} .
$$


To obtain a more compact writing for (3), we define in the following $\sigma^{*}(j)$ $=n+1-\sigma(j)$. We deduce from the definition of $U^{\Omega}$ and a well-known result in convex optimization that $\max _{p \in U^{\Omega}} \sum_{j \in \mathcal{J}} C_{j}(\sigma, p)=\sum_{j \in \mathcal{J}} \sigma^{*}(j) \bar{p}_{j}+$ $\Omega \sqrt{\sum_{i \in \mathcal{J}} \sum_{j \in \mathcal{J}} \sigma^{*}(i) \sigma^{*}(j) \hat{p}_{i} \hat{p}_{j} \Sigma_{i j}}$. We prove below that problem

$$
\min _{\sigma \in P(n)} \sum_{j \in \mathcal{J}} \sigma^{*}(j) \bar{p}_{j}+\Omega \sqrt{\sum_{i \in \mathcal{J}} \sum_{j \in \mathcal{J}} \sigma^{*}(i) \sigma^{*}(j) \hat{p}_{i} \hat{p}_{j} \Sigma_{i j}}
$$

is $\mathcal{N} \mathcal{P}$-hard for positive semi-definite matrices $\Sigma$. Strictly speaking, Problem (4) is more general that Problem 1||$U_{p}^{\Omega} \mid \sum_{j} w_{j} C_{j}$ because the former allows $\Sigma$ to a be a singular matrix, in which case $U^{\Omega}$ is not defined. However, one can show that both problems are equivalent by perturbing any singular matrix by a very small term to make it positive definite, see [7] for details.

Theorem 2.1 Problem 1||$U_{p}^{\Omega} \mid \sum_{j} w_{j} C_{j}$ is $\mathcal{N} \mathcal{P}$-hard.

Proof. Let $p^{1}$ and $p^{2}$ be two arbitrary vectors in $\mathbb{R}_{+}^{n}$. Yang and Yu [14] proved that the problem

$$
\min _{\sigma \in P(n)} \max _{p \in\left\{p^{1}, p^{2}\right\}} \sum_{j \in \mathcal{J}} C_{j}(\sigma, p)
$$

is $\mathcal{N} \mathcal{P}$-hard. We prove below that Problem (5) reduces to (4) by appropriate choices of $\bar{p}, \hat{p}, \Sigma$ and $\Omega$. Specifically, for each $j \in \mathcal{J}$, we let $\bar{p}_{j}=\frac{p_{j}^{1}+p_{j}^{2}}{2}$ and $\hat{p}_{j}=\frac{p_{j}^{1}-p_{j}^{2}}{2}$, and define $\Omega=1$ and $\Sigma_{i j}=1$ for each $i, j \in \mathcal{J}$. Consider any $\sigma \in P(n)$. The objective function of (4) becomes

$$
\begin{aligned}
& \sum_{j \in \mathcal{J}} \sigma^{*}(j) \frac{p_{j}^{1}+p_{j}^{2}}{2}+\sqrt{\sum_{i \in J} \sum_{j \in \mathcal{J}} \sigma^{*}(i) \sigma^{*}(j) \frac{p_{i}^{1}-p_{i}^{2}}{2} \frac{p_{j}^{1}-p_{j}^{2}}{2}} \\
= & \sum_{j \in \mathcal{J}} \sigma^{*}(j) \frac{p_{j}^{1}+p_{j}^{2}}{2}+\sqrt{\left(\sum_{j \in \mathcal{J}} \sigma^{*}(j) \frac{p_{j}^{1}-p_{j}^{2}}{2}\right)^{2}} \\
= & \sum_{j \in \mathcal{J}} \sigma^{*}(j) \frac{p_{j}^{1}+p_{j}^{2}}{2}+\left|\sum_{j \in \mathcal{J}} \sigma^{*}(j) \frac{p_{j}^{1}-p_{j}^{2}}{2}\right| \\
= & \sum_{j \in \mathcal{J}} \sigma^{*}(j) \frac{p_{j}^{1}+p_{j}^{2}}{2}+\max \left\{\sum_{j \in \mathcal{J}} \sigma^{*}(j) \frac{p_{j}^{1}-p_{j}^{2}}{2}, \sum_{j \in \mathcal{J}} \sigma^{*}(j) \frac{p_{j}^{2}-p_{j}^{1}}{2}\right\} \\
= & \max \left\{\sum_{j \in \mathcal{J}} \sigma^{*}(j) p_{j}^{1}, \sum_{j \in \mathcal{J}} \sigma^{*}(j) p_{j}^{2}\right\}=\max _{p \in\left\{p^{1}, p^{2}\right\}} \sum_{j \in \mathcal{J}} C_{j}(\sigma, p),
\end{aligned}
$$


proving the result.

\section{Integer programming formulations}

Next, we introduce two classical mathematical formulations for the problem.

Precedence formulation Let $x_{i j}$ be a binary variable equal to 1 if and only if job $i$ is scheduled prior to job $j$. We obtain the following formulation:

$$
\begin{aligned}
\underset{x \in\{0,1\}^{n^{2}}}{\operatorname{minimize}} & \left(\max _{p \in U} \sum_{i \in \mathcal{J}} \sum_{j \in \mathcal{J}} p_{i} w_{j} x_{i j}\right) \\
\text { s.t. } & x_{i j}+x_{j i}=1, \quad i, j \in \mathcal{J}, i \neq j \\
& x_{i j}+x_{j k} \leq x_{i k}+1, \quad i, j, k \in \mathcal{J} \\
& x_{i i}=1, \quad i \in \mathcal{J} .
\end{aligned}
$$

Using classical techniques, the objective function (6) can be rewritten as:

$U^{\Gamma}$ : The objective function (6) is replaced by $\sum_{i \in \mathcal{J}} \sum_{j \in \mathcal{J}} \bar{p}_{i} w_{j} x_{i j}+\Gamma z_{0}+$

$\sum_{i \in \mathcal{J}} z_{i}$, where $z_{0}$ and $z_{i}$ are $n+1$ additional non-negative variables that satisfy the additional constraints $z_{0}+z_{i} \geq \hat{p}_{i} \sum_{j \in \mathcal{J}} w_{j} x_{i j}$ for each $i \in \mathcal{J}$, leading to a mixed-integer linear programming reformulation.

$U^{\Omega}$ : The objective function (6) is replaced by $\sum_{i \in \mathcal{J}} \sum_{j \in \mathcal{J}} \bar{p}_{i} w_{j} x_{i j}+\left\|\tilde{x}^{T} \Sigma \tilde{x}\right\|_{2}$ where $\tilde{x}_{i}=\sum_{j \in \mathcal{J}} \hat{p}_{i} w_{j} x_{i j}$, leading to a mixed-integer second order cone programming reformulation.

Whenever $\Gamma=1$, one readily verifies that there exists an optimal solution with $z_{j}=0$ for each $j \in \mathcal{J}$. We obtain the objective function $\sum_{i \in \mathcal{J}} \sum_{j \in \mathcal{J}} \bar{p}_{i} w_{j} x_{i j}+$ $z_{0}$ combined with the additional constraints $z_{0} \geq \hat{p}_{i} \sum_{j \in \mathcal{J}} w_{j} x_{i j}$ for each $i \in \mathcal{J}$.

Assignment formulation Let $y_{i j}$ be a binary variable equal to 1 if and only if job $i$ is scheduled in position $j$. We obtain the following formulation:

$$
\begin{aligned}
\underset{y \in\{0,1\}^{n^{2}}}{\operatorname{minimize}} & \left(\max _{p \in U} \sum_{i \in \mathcal{J}} \sum_{j \in \mathcal{J}} \sum_{k \in \mathcal{J}} \sum_{l=1}^{j} p_{i} w_{k} y_{i j} y_{k l}\right) \\
\text { s.t. } & \sum_{i \in \mathcal{J}} y_{i j}=1, \quad j \in \mathcal{J} \\
& \sum_{j \in \mathcal{J}} y_{i j}=1, \quad i \in \mathcal{J}
\end{aligned}
$$


which is a special case of the quadratic assignment problem. We linearize the products between variables $y_{i j}$ and $y_{k l}$ by introducing new real variables $z_{i j k l}$ satisfying $z_{i j k l} \leq y_{i j}, z_{i j k l} \leq y_{k l}$, and $z_{i j k l} \geq y_{i j}+y_{k l}-1$. Then, the two classical reformulations described for the precedence formulation can be used to reformulate the linearized objective function (10).

\section{Numerical experiments}

We compare the different formulations presented in the previous section for the two uncertainty sets. We solve the formulation using the aforementioned reformulation or using branch-and-cut algorithms, which is further detailed below.

Instances and implementation details The instances were randomly generated as follows. For each $j \in \mathcal{J}$, the processing times $\bar{p}_{j}, \hat{p}_{j}$, and weight $w_{j}$ have been uniformly generated in the intervals $[1,2 n],[1, n]$ and $[1, n]$, respectively. The algorithms are coded in Julia language, using the package Jump [8], and solved by Gurobi 6.5. The experiments are carried out on a computer equipped with a $\mathrm{CPU}$ at $2.67 \mathrm{GHz}$ and $125 \mathrm{~GB}$ of memory. A time limit of 1800 seconds was imposed for each instance and all solution times are reported in seconds.

Value-at-risk The values of $\Gamma$ and $\Omega$ have been computed using the probabilistic bounds provided in [5] and [2], respectively. Specifically, these values ensure that the optimal solution of (2) provides a conservative approximation to the value-at-risk optimization problem $\min _{\sigma \in P(n)} \operatorname{VaR}_{\epsilon}\left[\max _{p \in U} \sum_{j \in \mathcal{J}} w_{j} C_{j}(\sigma, p)\right]$, whenever $p$ is any random vector and

$$
\operatorname{VaR}_{\epsilon}\left[\max _{p \in U} \sum_{j \in \mathcal{J}} w_{j} C_{j}(\sigma, p)\right]=\inf \left\{t: P\left(\max _{p \in U} \sum_{j \in \mathcal{J}} w_{j} C_{j}(\sigma, p) \geq t\right) \leq \epsilon\right\}
$$

see also $[10,11]$ for more details on the relation between $U^{\Gamma}$ and probabilistic constraints $^{4}$. Given a probability $\left.\epsilon \in\right] 0,1[$, we obtain (see [2]) that $\Omega(\epsilon)=$ $\sqrt{-2 \ln \epsilon}$ while the formula is more complex for $\Gamma$, for which we refer to [5].

\footnotetext{
$\overline{4}$ Notice that the less conservative model proposed in $[10,11]$ cannot be used here to reduce the conservatism of $U^{\Gamma}$ because all coefficients of $p$ are non-zero in the objective function.
} 
Branch-and-cut algorithm In addition to the reformulations described in Section 3, we solve the precedence formulation via branch-and-cut algorithms in the line of [3]. Specifically, the algorithm considers a restricted master problem formed by constraints (7)-(9), with the additional constraint $\gamma \geq$ $\sum_{i \in \mathcal{J}} \sum_{j \in \mathcal{J}} p_{i} w_{j} x_{i j}$, and minimizes variable $\gamma$. Then, at each integer node, we solve the separation problem

$$
z=\max _{p \in U}\left(\sum_{i \in \mathcal{J}} \sum_{j \in \mathcal{J}} p_{i} w_{j} x_{i j}^{*}\right),
$$

where $x^{*}$ and $\gamma^{*}$ denote the current values of $x$ and $\gamma$, respectively. If $z>\gamma^{*}$, we add the cutting plane $\gamma \geq \sum_{i \in \mathcal{J}} \sum_{j \in \mathcal{J}} p_{i}^{*} w_{j} x_{i j}$ where $p^{*} \in U$ is an optimal solution of (11). Notice that for $U^{\Gamma}$ and $U^{\Omega}$, the separation problem amounts to sort a vector and to compute an $L 2$-norm, respectively (see [3] for details).

Results Table 1 compares the precedence formulation with the assignment formulation using dualized reformulations. The table reports the times spent solving the linear programming relaxation as well as the full problems, and the root gaps. The results shows that the assignment formulation is weaker and takes a longer time to be solved than the precedence formulation. Table 2 compares the classical dualization with the specialized reformulation proposed above when $\Gamma=1$. The table seems to indicate that both formulations behave similarly with Gurobi. Table 3 compares dualizations with branch-and-cut algorithms for the two uncertainty sets. It appears that the dualization is faster for $U^{\Gamma}$ while the branch-and-cut algorithm outperforms the dualization for $U^{\Omega}$. Table 4 finally compares the solution costs of the two models for the same levels of probability. The results show that the approximation of the value-at-risk provided by $U^{\Omega}$ is cheaper than the one provided by $U^{\Gamma}$.

\section{Acknowledgements}

We would like to thank Janis Kurtz for providing us with reference [7].

\section{References}

[1] H. Aissi, C. Bazgan, and D. Vanderpooten. Min-max and min-max regret versions of combinatorial optimization problems: A survey. European Journal of Operational Research, 197(2):427-438, 2009. 


\begin{tabular}{cc|ccc|ccc}
\hline$n$ & $\Gamma$ & \multicolumn{3}{|c|}{ Precedence formulation } & \multicolumn{3}{|c}{ Assignment formulation } \\
& & time LP & time IP & Gap & time LP & time IP & Gap \\
\hline 5 & 4.12 & 0.005 & 0.002 & 0 & 2.418 & 0.0056 & 100 \\
10 & 5.226 & 0.014 & 0.006 & 0.294 & 292.193 & 0.171 & 100 \\
15 & 6.11 & 0.137 & 0.0219 & 0.142 & 1800 & 1.144 & 100 \\
20 & 6.854 & 0.670 & 0.075 & 0.344 & 1800 & 4.437 & 100 \\
\hline
\end{tabular}

Table 1

Comparison of the precedence formulation and the assignment formulation for $U^{\Gamma}$

\begin{tabular}{cc|cc}
\hline$n$ & $\Gamma$ & Classical dualization & Specialized reformulation \\
\hline 50 & 1 & 10.8840 & 5.5348 \\
60 & 1 & 17.2279 & 24.9224 \\
70 & 1 & 18.3319 & 16.0779 \\
80 & 1 & 14.5652 & 15.4387 \\
90 & 1 & 26.8292 & 32.3861 \\
100 & 1 & 32.8103 & 26.9855 \\
110 & 1 & 75.4261 & 89.5468 \\
120 & 1 & 86.2200 & 80.9256 \\
130 & 1 & 100.4396 & 100.9902 \\
140 & 1 & 108.2194 & 135.1086 \\
150 & 1 & 145.8819 & 157.2395 \\
200 & 1 & 427.3675 & 489.7448 \\
\hline
\end{tabular}

Table 2

Comparisons of the solution times of the two formulations under the uncertainty set $U^{\Gamma}$ with $\Gamma=1$

[2] A. Ben-Tal and A. Nemirovski. Robust solutions of linear programming problems contaminated with uncertain data. Math. Program., 88(3):411-424, 2000 .

[3] D. Bertsimas, I. Dunning, and M. Lubin. Reformulation versus cutting-planes for robust optimization. Computational Management Science, 13(2):195-217, 2016 .

[4] D. Bertsimas and M. Sim. Robust discrete optimization and network flows. Math. Program., 98(1-3):49-71, 2003.

[5] D. Bertsimas and M. Sim. The price of robustness. Operations Research, $52(1): 35-53,2004$.

[6] M. Bougeret, A. Pessoa, and M. Poss. Robust scheduling with budgeted uncertainty. Working paper, 2016. 


\begin{tabular}{|c|c|c|c|c|c|}
\hline \multirow[t]{2}{*}{$n$} & \multirow[t]{2}{*}{$\epsilon$} & \multicolumn{2}{|c|}{$U^{\Gamma}$} & \multicolumn{2}{|c|}{$\overline{U^{\Omega}}$} \\
\hline & & Dualization & $\mathrm{B} \& \mathrm{C}$ & Dualization & $\mathrm{B} \& \mathrm{C}$ \\
\hline \multirow{3}{*}{50} & 0.01 & 5.3192 & 17.7836 & 1756.1289 & 14.4455 \\
\hline & 0.05 & 2.6841 & 10.9062 & 1600.961 & 16.725 \\
\hline & 0.1 & 2.8941 & 12.0554 & 1333.4534 & 5.0623 \\
\hline \multirow{3}{*}{60} & 0.01 & 44.5364 & 81.8779 & 1800 & 24.6955 \\
\hline & 0.05 & 31.7562 & 73.0690 & 1800 & 17.7427 \\
\hline & 0.1 & 15.0364 & 26.9620 & 1800 & 16.5177 \\
\hline \multirow{3}{*}{70} & 0.01 & 36.6147 & 91.0086 & 1800 & 48.3793 \\
\hline & 0.05 & 13.1456 & 42.1255 & 1800 & 30.1910 \\
\hline & 0.1 & 3.8862 & 24.3906 & 1800 & 23.7368 \\
\hline \multirow{3}{*}{80} & 0.01 & 47.1291 & 130.2417 & 1800 & 81.7222 \\
\hline & 0.05 & 36.2294 & 130.1191 & 1800 & 52.5677 \\
\hline & 0.1 & 8.8290 & 21.0278 & 1800 & 44.6953 \\
\hline \multirow{3}{*}{90} & 0.01 & 27.2781 & 181.6671 & 1800 & 127.6601 \\
\hline & 0.05 & 27.7035 & 181.4539 & 1800 & 93.1146 \\
\hline & 0.1 & 27.2781 & 170.6671 & 1800 & 73.1298 \\
\hline \multirow{3}{*}{100} & 0.01 & 55.3856 & 292.3421 & 1800 & 232.755 \\
\hline & 0.05 & 21.8976 & 89.7856 & 1800 & 99.1304 \\
\hline & 0.1 & 19.6913 & 83.9951 & 1800 & 82.1937 \\
\hline \multirow{3}{*}{110} & 0.01 & 33.1948 & 253.8273 & 1800 & 107.6149 \\
\hline & 0.05 & 30.1278 & 186.2178 & 1800 & 117.9020 \\
\hline & 0.1 & 26.5111 & 101.226 & 1800 & 147.3104 \\
\hline \multirow{3}{*}{120} & 0.01 & 46.9315 & 247.8843 & 1800 & 226.3862 \\
\hline & 0.05 & 43.7567 & 199.9976 & 1800 & 115.1185 \\
\hline & 0.1 & 44.61242 & 206.2942 & 1800 & 193.9772 \\
\hline \multirow{3}{*}{130} & 0.01 & 81.1503 & 331.1503 & 1800 & 254.1678 \\
\hline & 0.05 & 59.3245 & 299.3246 & 1800 & 483.1201 \\
\hline & 0.1 & 62.1288 & 202.1288 & 1800 & 220.4472 \\
\hline \multirow{3}{*}{140} & 0.01 & 110.1452 & 523.3927 & 1800 & 523.0023 \\
\hline & 0.05 & 103.6665 & 403.6665 & 1800 & 549.0178 \\
\hline & 0.1 & 95.1212 & 431.2345 & 1800 & 289.8857 \\
\hline \multirow{3}{*}{150} & 0.01 & 132.7827 & 732.617 & 1800 & 429.1916 \\
\hline & 0.05 & 112.4356 & 639.4243 & 1800 & 438.6716 \\
\hline & 0.1 & 112.7711 & 572.771 & 1800 & 737.3711 \\
\hline \multirow{3}{*}{200} & 0.01 & 880.156 & 1800 & 1800 & 1219.8972 \\
\hline & 0.05 & 511.3178 & 1800 & 1800 & 1395.2189 \\
\hline & 0.1 & 580.443 & 1800 & 1800 & 1618.8333 \\
\hline
\end{tabular}

Table 3

Solution times for uncertainty sets $U^{\Gamma}$ and $U^{\Omega}$ 


\begin{tabular}{cc|c|c}
\hline $\mathrm{n}$ & $\epsilon$ & $U^{\Gamma}$ & $U^{\Omega}$ \\
\hline & 0.01 & $1.236124004 \mathrm{e} 6$ & $1.0973274217 \mathrm{e} 6$ \\
50 & 0.05 & $1.3787137 \mathrm{e} 6$ & $1.2001925699 \mathrm{e} 6$ \\
& 0.1 & $1.2636933399 \mathrm{e} 6$ & $1.1033971647 \mathrm{e} 6$ \\
\hline \multirow{3}{*}{100} & 0.01 & $1.643406456 \mathrm{e} 7$ & $1.47192456036 \mathrm{e} 7$ \\
& 0.05 & $2.010210435 \mathrm{e} 7$ & $1.8023336381 \mathrm{e} 7$ \\
& 0.1 & $1.898526685 \mathrm{e} 7$ & $1.70664998303 \mathrm{e} 7$ \\
\hline \multirow{3}{*}{150} & 0.01 & $8.0721378208 \mathrm{e} 7$ & $7.18727789599 \mathrm{e} 7$ \\
& 0.05 & $8.441931962 \mathrm{e} 7$ & $7.59088424515 \mathrm{e} 7$ \\
& 0.1 & $7.31019883599 \mathrm{e} 7$ & $6.54991746364 \mathrm{e} 7$ \\
\hline \multirow{3}{*}{200} & 0.01 & $2.8539763498 \mathrm{e} 8$ & $2.4891821698121 \mathrm{e} 8$ \\
& 0.05 & $2.31240831376 \mathrm{e} 8$ & $2.077655828647 \mathrm{e} 8$ \\
& 0.1 & $2.5827205682 \mathrm{e} 8$ & $2.372011370971 \mathrm{e} 8$ \\
\hline
\end{tabular}

Table 4

Solution costs for models $U^{\Gamma}$ and $U^{\Omega}$

[7] C. Buchheim. Robuste optimierung, 2014. http://www.mathematik.tudortmund.de/lsv/teaching/robopt/Skript.pdf.

[8] I. Dunning, J. Huchette, and M. Lubin. Jump: A modeling language for mathematical optimization. CoRR, abs/1508.01982, 2015.

[9] R. L. Graham, E. L. Lawler, J. K. Lenstra, and A. R. Kan. Optimization and approximation in deterministic sequencing and scheduling: a survey. Annals of discrete mathematics, 5:287-326, 1979.

[10] M. Poss. Robust combinatorial optimization with variable budgeted uncertainty. 4OR, 11(1):75-92, 2013.

[11] M. Poss. Robust combinatorial optimization with variable cost uncertainty. European Journal of Operational Research, 237(3):836-845, 2014.

[12] W. E. Smith. Various optimizers for single-stage production. Naval Research Logistics Quarterly, 3(1-2):59-66, 1956.

[13] B. Tadayon and J. C. Smith. Algorithms and complexity analysis for robust single-machine scheduling problems. Journal of Scheduling, 18(6):575-592, 2015.

[14] J. Yang and G. Yu. On the robust single machine scheduling problem. Journal of Combinatorial Optimization, 6(1):17-33, 2002. 